\title{
Simulation of Gas Speed Measurement for Investigating Maxwell Distribution
}

\author{
Tugiyo Aminoto ${ }^{1}$, Rahma Dani ${ }^{2}$, Neneng Lestari ${ }^{3}$, Cicyn Riantoni ${ }^{4}$ \\ \{tugiyo@unja.ac.id ${ }^{1}$, radanicev@yahoo.com² ${ }^{2}$ neneng14lestari@gmail.com ${ }^{3}$ \}
}

Universitas Jambi, Indonesia ${ }^{1,2,3,4}$

\begin{abstract}
Teaching and learning which mainly based on conventional media offers limited resources in exploring a comprehensive concept of statistical physics phenomena. As a consequence, it needs to incorporate a computer simulation that has superiority in visualizing a wide range of complicated and abstract physics concepts. This study aims to develop a computer simulation of gas speed measurement as a learning medium on the subject of Maxwell distribution. Measurement of very small and un-naked eye gas particles requires special techniques and tools that involve a complicated process of selecting particles and measuring their speed. For learning purposes, this measurement process needs to be done with computer simulation. This research uses the ADDIE model which includes analysis, design, development, implementation and evaluation. The results of the simulation show conformity with Maxwell's speed distribution graph which was obtained theoretically and need to apply to enhance the quality of teaching of this subject.
\end{abstract}

Keywords: Computer Simulation, Gas Speed Measurement, Maxwell Distribution

\section{Introduction}

Teaching and learning that mainly focus on reading texts and deriving formulas will cause difficulties for students to understand physical phenomena. College students don't ordinarily think that it's hard to utilize arithmetic and formulae, which isn't astonishing thinking about that their guidance centers around these segments [1]. Customary guidance in material science courses concentrated on critical thinking disregards significant procedural objectives and is typically concerned for the most part with numerical activities [2]. This Traditional for the most part prompts poor accomplishment as far as creating abilities and strategies related to critical thinking [3]. Analyzing the impact of using simulation-based materials, it found that the number of the experimental students who used expert-type scientific procedures (qualitative analysis of the problem, making hypotheses, and analysis of results) was increased and there was a decrease for the number of those whose habits based solely on mathematical equations [4].

The rapid development of information technology and computers and the paradigm shift in learning that is increasingly active, creative and fun raises challenges as well as solutions. The impact is that education practitioners and researchers have used ICT-based learning media a lot because it is very useful for improving the quality of science learning. Technology has shown an incredible potential to improve learning and are generally consolidated into instructional exercises to enable instructors to accomplish different learning objectives. Going for various learning objectives, innovations, for the most part, grasp different capacities and highlights [5]. 
In discussing simulation, it is necessary to review the system and model. The model is a description of several systems that are intended to predict what happens if certain actions are taken. Each model is useful for fatherly simplifying and idealizing the problem. For a model to be useful, it is important that, given a fairly limited set of descriptors, all relevant behaviors and properties can be determined in a practical way: analytically, numerically, or by moving the model with certain (usually random) inputs and observing the appropriate outputs. This process is called simulation [6].

Virtual simulations can be an alternative or a supplement to the experimental activities. If high costs become an issue in the procurement of laboratory equipment, especially for developing universities, virtual laboratories can also be a solution. In cases where students lag behind or cannot participate in practical work for various reasons, there is often difficulty in doing practical work for just one or two people. Virtual labs can be available anytime and anywhere, offer students more information, and offer students the opportunity to work at their own pace while exploring difficult or interesting concepts. In addition, it is very helpful for students who are physically unable to attend classes [7]. It mention that there are various other advantages that can be obtained with a computer simulation method: more interactive and effective, increase understanding, reduced equipment needs, available at any time from any place, offered more information to students, and offered students the opportunity to work at their own pace $[7,8]$.

A study to see whether there were differences in effectiveness between laboratory practices and practicum and computer simulations has been conducted. This research involved 224 students from two major universities namely Auburn University and Penn State University to investigate the effectiveness of the learning process for students who use a computer simulations lab and the learning process for students who do so in an ordinary or traditional laboratory. Data analysis at both universities shows that the learning approach is both effective [7]. Another research also conducted on the topic of improving the quality of physics learning processes by using computer simulations on the problem of storm phenomena. Analysis of student learning outcomes and interest increases after learning by using the simulator. The results of this study indicate that by using a storm simulator, it can improve student learning processes so that on the one hand students understand the storm phenomenon, and on the other hand students assimilate with better physics ideas. Computer technology is a positive supplement to bridge the gap between education and the world of technology [9]. The same topic of research is a study of physics learning with simulations in the case of teacher-centered and student-centered learning. The results of this study indicate that the use of Physlets simulations in the classroom on teachercentered learning can be more effective than using Physlets simulations in student-centered computer laboratories for conceptual understanding [10].

On the subject of Maxwell's speed distribution, students study the characteristics of ideal gas particle speeds. The velocity distribution of the gas particles is obtained theoretically by equation (1) below [11]:

$$
N(v)=N(E) \frac{d E}{d v}=\frac{2 N}{\sqrt{\pi}(k T)^{3 / 2}} \sqrt{\frac{m v^{2}}{2}} e^{-m v^{2} / 2 k T} m v=N \sqrt{\frac{2}{\pi}}\left(\frac{m}{k T}\right)^{3 / 2} v^{2} e^{-m v^{2} / 2 k T}
$$

This problem is related to the measurement of very small and invisible gas particles that require special techniques and tools (involving the process of selecting particles and measuring their speed). In the real lab the measurement is e.g. conducted by using two moving discs as shown on Figure 1: 


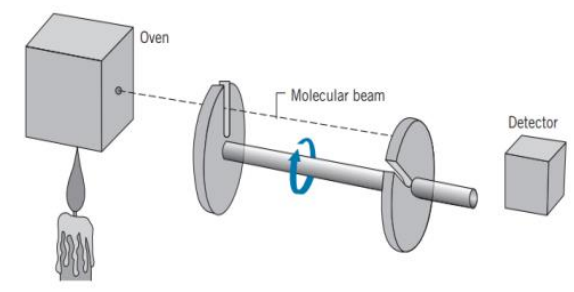

Fig. 1. Gas speed measurement device [11]

The picture shows that the gas particles coming out of the oven hole will go through disk 1 and disk 2 with distance L. The gap2 in disk 1 and 2 is placed with angle $\alpha$ and both are rotated with angular velocity $\omega$. Furthermore, particles that pass through the 2 disks will be able to reach a detector; otherwise it will not reach the detector. The equation that applies is $\mathrm{L} / \mathrm{v}=\alpha / \omega$ with $\mathrm{L}$ and $\alpha$ are fixed. Therefore, the speed of a particle that can reach the detector is proportional to $\mathrm{w}$, equation (2) :

$$
\mathrm{v}=\mathrm{k} \omega ; \mathrm{k}=\mathrm{a} \text { constant }
$$

The process of measuring particle velocity is conducted through the particle selection process so it can be known how many particles have a certain speed $\mathrm{v}$ in time $\Delta \mathrm{t}$. This process really needs to be understood by students in order to really have an in-depth understanding of obtaining the Maxwell speed distribution based on the experiment. Abstract concepts require a special learning approach. The use of computer simulation media can visualize abstract things more concretely. The simulation uses mathematical descriptions or models, from real systems in the form of computer programs. Computer simulations are used to study the dynamic behavior/characteristics of objects or systems that cannot be easily or safely learned in real life. Giving input to the model with a certain value and observing its output, this process is called the simulation process [6]. The use of computer simulations is a solution to the absence or limitations of practicum tools and learning media due to facilities and funding factors [7].

Computational verification of the Maxwell distribution has been carried out using cellular automata [13]. The results are in accordance with the theory and measurement results in the lab. However, the simulation does not emphasize the visualization aspect so that it is still not suitable to be used as a medium of learning in the classroom. In addition, the measurement of the gas rate is directly carried out by the computer program itself, the users only receive the measurement result and have no role so they will not gain the opportunity of learning by doing.

Not all physics labs have gas velocity gauges and the unavailability of gas velocity measurement simulations is an important factor for this research. Learning media in the form of real objects or virtual simulations are really needed by teachers and students in learning physics. This simulation is also useful to obtain an in-depth picture of the gas velocity measurement process that has been done in a lab that has been able to do it. For the purposes of enhancing learning media, the development of computer simulations that can visualize its mechanism is very important. 


\section{Method}

A model that is often used in development research is the ADDIE model. This model has a sequence of activities that are systematic and complete in an effort to solve the problem of making and developing a product or model. This research is development research (R\&D) with the aim of producing products in the form of learning media based on Adobe Flash (using action script 3.0). The model used is ADDIE (Analyze, Design, Development, Implementation, and Evaluation) [14].

In the analysis phase, identification of the difficulties of prospective teacher students was carried out in understanding the concept of gas velocity measurement to obtain the MaxwellBoltzmann distribution. Furthermore, a literature study is carried out on the existing simulation media to find out its shortcomings and followed by the design stage to obtain a more appropriate product. Initial designs continue to be developed and improved based on the stages of implementation and evaluation.

\section{Result and Discussion}

prospective physics teachers who had taken physics courses. It suggests that $62 \%$ of respondents having difficulties in understanding the process of gas speed measurement and expressed interest. They felt helped if there were learning media in the form of computer simulations. Their reason was by mainly listening to lecturers' explanations, reading and discussing Maxwell's velocity distribution material, students find it difficult to understand the process of measuring gas velocity let alone theoretically decrease it. On the other hand, the number of respondents who expressed doubt was $38 \%$. Their reason was that they were more optimistic if the original device is used compared to computer simulations. In this case, the respondent did not realize how difficult the measurement was when using the real tool and also the availability factor of this expensive tool. The results of the curriculum analysis are: the understanding of Maxwell's velocity distribution needs to be supported by media whose visual superiority in increasing HOTS and critical thinking skills of students.

1. After analyzing the needs and the shortcomings of existing simulation, the programming algorithm is constructed as follow:

2. The input is moving particles that come out from the 2D-box's hole. The speeds of these moving particles are randomly varied due to the collision process in the box.

3. The size of particles are identic, small but still easy to be observed by the user

4. For simplification, a 2-dimensional approach will be used: disk 1, disk 2 and the gaps will each be drawn in the form of 2D (top view).

5. In $2 \mathrm{D}$, the rotation of the disk is sufficiently represented by linear movement of the gap while the disks remain in its place.

6. The rotating motion of the discs and the gaps are considered as linear-vertical motion (up and down) with speed of v.

7. The length of time of simulation is in the range of minutes.

With the simulation shown on Figure 2, students can observe particles coming out of the oven and passing 2 rotating discs. Particles that hit the disc will bounce and those that hit the slit will go to disk 2 . The same event will occur only particles that hit the gap will be able to go 
to the detector. Only particles with a certain rate can enter into the disc gap. Particles that are too slow or too fast will crash into the disk and bounce off. This simulation accentuates visualization, unlike the previous simulations which have no visualization so it is less suitable to be used as a medium of learning in the classroom [13]. Moreover, In deriving the Maxwell speed distribution formula it is related to the calculation of the number of molecules that have energy between $\mathrm{E}$ and $\mathrm{E}+\mathrm{dE}$ in gas or speed $\mathrm{v}$ and $\mathrm{v}+\mathrm{dv}$. The term $\mathrm{dv}$ expresses the speed interval that theoretically arises. The mathematical and abstract calculations of $\mathrm{dv}$ need to be visualized and concretized with a computer simulation as developed in this simulation. Therefore, teaching and learning which aided by such a simulation approach may significantly help to concretize the abstract and complex phenomena in science education. Thus simulation assists students to learn more easily and more effectively [12].

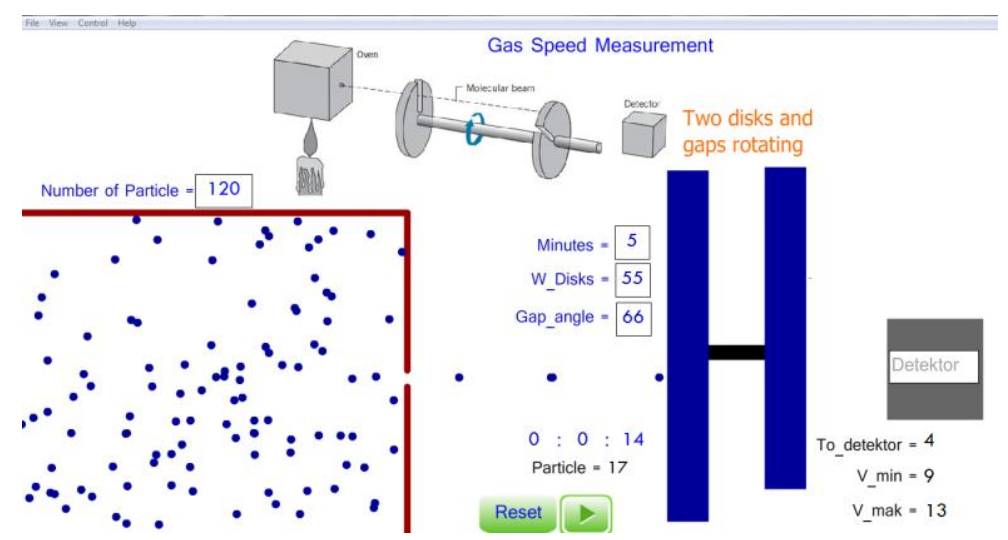

Fig. 2. Screen-shoot of simulation.

The most interesting finding from this simulation is the speed of the particle entering the detector is not a single speed but can be more than one speed (has an interval dv). According to equation (2), $\mathrm{v}=\mathrm{k} \omega$, it implies one value of disc speed only corresponds to one value of particle speed. This fact will trigger curiosity and encourage students to think critically when comparing theories with simulations. Students will find that this happened because in the real measurement (and also in the simulation) the slot of the disc has a certain value of width which cause the possibility of being passed by several particles whose slightly different speeds as Figure 3 shown:

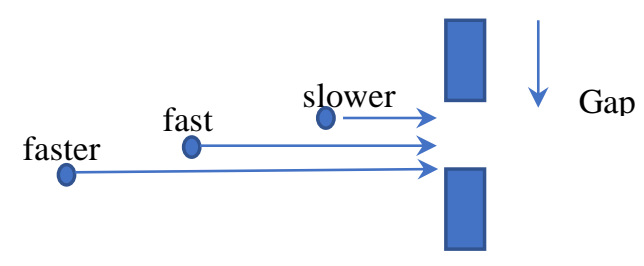

Fig. 3. Possibility of three particles with different speed when passing the moving gap.

Even though this speed variation creates difficulties in determining which speed will be used, this problem is overcome by adding a programming code that can record all the particle speeds that enter the detector and then determine the value of the min and the max. In data 
processing, average speeds are used in the calculation to draw the graph. Such computer simulation, as point out by Calik [15], can empower the understudies to extend their thinking experience, experience scientific phenomena, and conduct investigations to perform scientific inquiry.

In developing simulations, before analyzing the output (recorded by the detector), checking the input characteristics needs to be done to determine the consistency of the output with the inputs. In this case, the input (the rate of particles coming out of the oven hole) in theory has a normal distribution pattern.

On Figure 4 can be seen that the particle speed profile (coming out of the oven hole) is in the form of a normal distribution but it has fluctuated which is caused by the nature of randomness of the system. The following Table 1 is the simulation results obtained from disks speed variation with the angle of the slots of 70 .

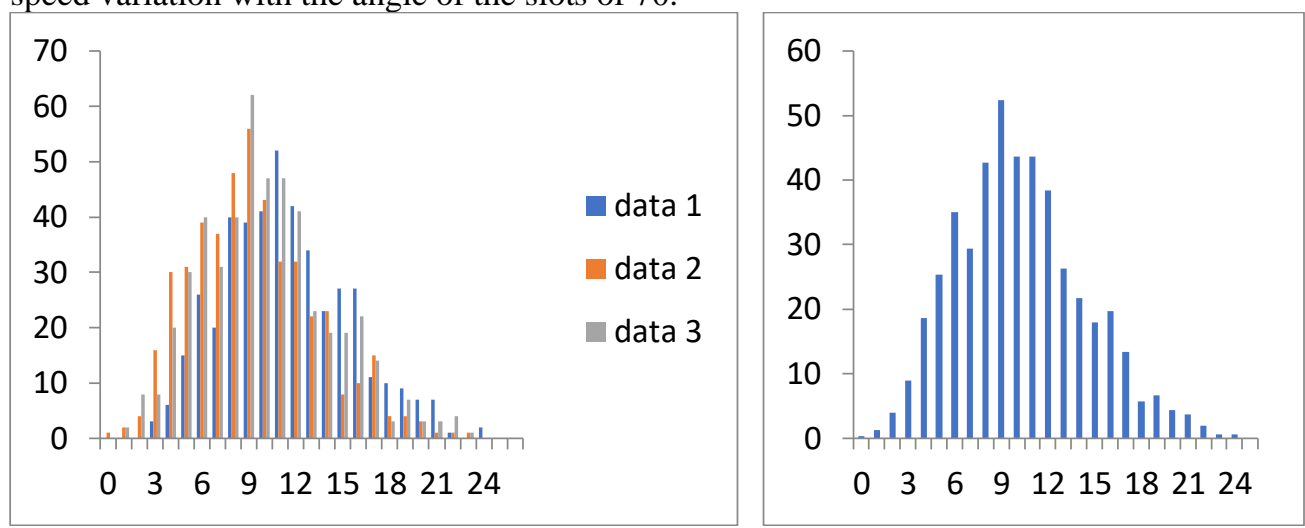

Fig.4. Speed fluctuation: particles escaped from the oven's hole (left) its average (right).

Table 1. Result of simulation (each variation take $t=5$ minutes).

\begin{tabular}{ccccccc}
\hline No & v_disk & v_min & v_max & v_average & $\Delta \mathrm{v}$ & $\begin{array}{c}\text { Number of particles } \\
\text { reached Detector }\end{array}$ \\
\hline 1 & 11 & 5 & 11 & 8 & 6 & 5 \\
2 & 22 & 5 & 17 & 11 & 12 & 6 \\
3 & 33 & 4 & 17 & 10,5 & 13 & 16 \\
4 & 44 & 7 & 20 & 13,5 & 13 & 19 \\
5 & 55 & 7 & 22 & 14,5 & 15 & 21 \\
6 & 66 & 8 & 20 & 14 & 12 & 16 \\
7 & 77 & 9 & 16 & 12,5 & 7 & 11 \\
8 & 88 & 12 & 23 & 17,5 & 11 & 7 \\
9 & 99 & 14 & 20 & 17 & 6 & 5 \\
\hline
\end{tabular}

The data is then graphed with Excel application as can be seen on Figure 5. 

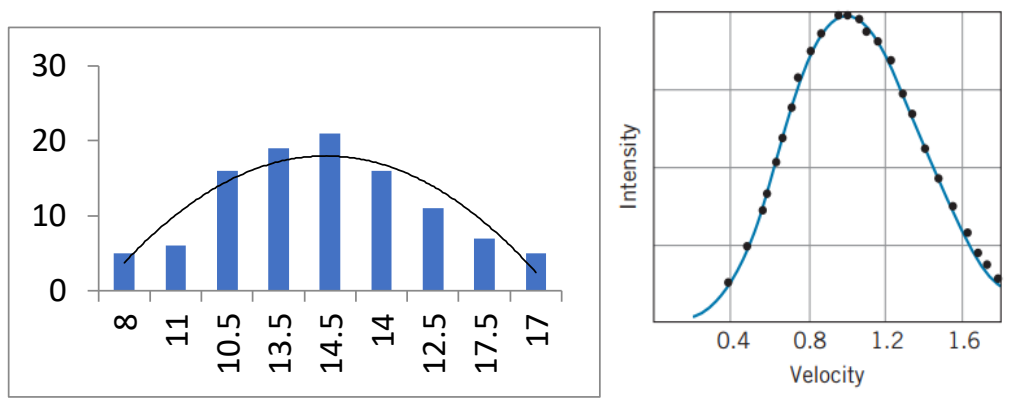

Fig. 5. Conformity degree of simulation results (left) and real lab experiment (right).

This gas speed measurement is deal with a random process if it is repeated it possibly show slight fluctuated results. The graphs of several trials of measurements have fluctuation trends as can be seen on Figure 6.
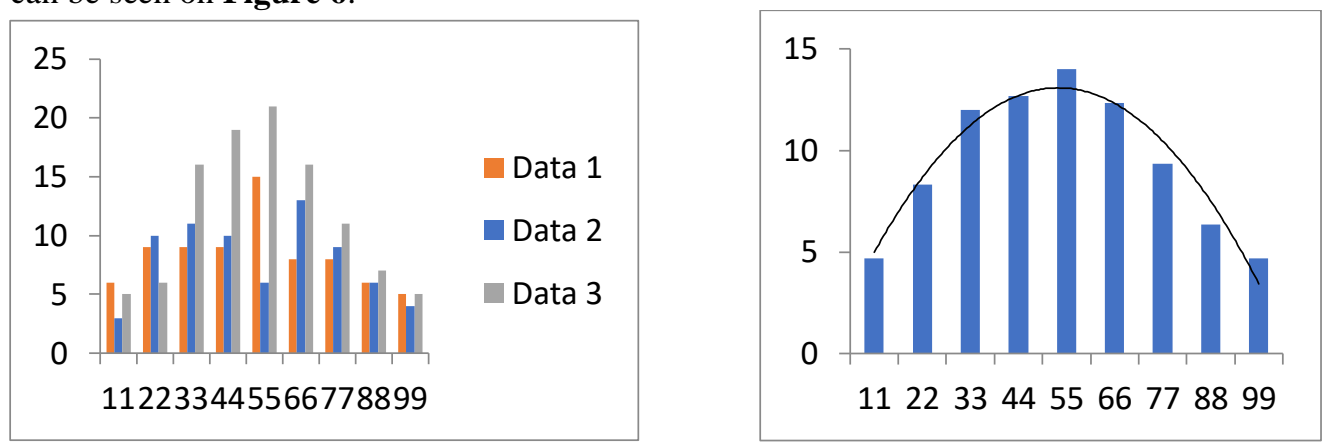

Fig. 6. Fluctuation on repeated measurement due to randomness (left) and its average (right).

The results of each data collection show a slightly different (there is a deviation in the distribution pattern of particle speeds which in theory it shows Maxwell distribution) but if some of the experimental results are accumulated or averaged it turns out that the results obtained are more in line with the Maxwell distribution pattern. Other factors that cause incompatibility with theoretical results are computational limitations, for example, due to particle size that cannot be as small as possible, system size and the number of particles that are still not large enough.

\section{Conclusion}

Computer simulation of gas speed measurement can be used as a learning medium in investigating and understanding Maxwell's speed distribution. It is interactive so students can adjust the duration of the simulation, the rotational speed of the disc and the angle between the gaps. The results of each data measurement show a slightly different pattern from its theory (due to randomness) but if some of the experimental results are accumulated it turns out that the results are in line with the Maxwell distribution pattern as obtained in theory. Furthermore, the results of the implementation to the students found that the majority of students expressed interest in using it and agreed that this media is very helpful in visualizing complex and abstract 
concepts. Teaching the Maxwell distribution concept is advised to use this simulation so that their students are more motivated and get more understanding because of the process of observation and experimentation in the simulation.

\section{References}

[1] Kim E., Pak S.J.: Students do not overcome conceptual difficulties after solving 1000 traditional problems. Am J Phys 70(7): pp. 759-765 (2002)

[2] Dervic D. et al .: Teaching Physics With Simulations: Teacher Centered Versus Student Centered Approaches. Journal of Baltic Science Education. Vol. 17 No. 2 (2018)

[3] Dancy M, Henderson, C.: Framework for articulating instructional practices and conceptions. Phys Rev Spec Top Phys Educ Res 3(1): pp. 010103 (2007)

[4] Ceberio M., Almudı M., Franco A.: Design and Application of Interactive Simulations in ProblemSolving in University-Level Physics Education. pp. 590-609. https://doi.org/10.1007/s10956-0169615-7 (2016)

[5] Zhang, L.: A meta-analysis method to advance design of technology-based learning tool: combining qualitative and quantitative research to understand learning in relation to technology-based learning tool: combining qualitative and quantitative research to understand learning in relation to different technology features. J Sci Educ Technol 23(1): pp. 145-159 (2013)

[6] Bratley, Fox, Schrage.: A Guide to Simulation. Springer (2011)

[7] Darrah M., et al.: Are Virtual Labs as Effective as Hands-on Labs for Undergraduate Physics? A Comparative Study at Two Major Universities. Journal Science Education Thecnol 23: pp. 803-814 (2014)

[8] David C. G., Elizabeth B., Gerald K., Pamela R.: Handbook of Games and Simulations in Teacher Education, AACE USA (2014)

[9] Munoz, Miriam M, et al.: Electrical Storm Simulation to Improve the Learning Physics Process. Journal Informatics in Education. Vol. 12 No 2: pp. 191-206 (2013)

[10] Dufresne R.J., Gerace, W.J.: Assessing to learn: formative assessment in physics instruction. Phys Teach 42: pp. 428-433 (2004)

[11] Krane, K.S.: Modern Physics 3rd Edition. John Wiley \& Sons, Inc (2012)

[12] Akpmar, E.: The use of interactive computer animations based on POE as a presentation tool in primary science teaching. J Sci Educ Technol 16(5): pp. 401-411 (2014)

[13] Khotimah S.N., Arif I., Liong T.H.: Lattice-Gas Automata for Numerical Experimental Verification of Maxwell-Boltzmann Distribution. Kontribusi Fisika Indonesia Vol. 12 No. 3 (2001)

[14] Branch, R. M.: Instructional Design: The ADDIE Approach. Springer (2009)

[15] Calik M., Ebenezer J., Ozsevgec T., Kucuk Z., Artun H.: Improving science student teachers' selfperceptions of fluency with innovative technologies and scientific inquiry abilities. J. Sci. Educ. Technol. 23(3): pp. 412-430 (2015) 